\title{
Laparoscopic Sleeve Gastrectomy with an Extensive Posterior Mobilization: Technique and Preliminary Results
}

\author{
Ralph P. M. Gadiot • Lacer Ulas Biter • Hans J. F. Zengerink • \\ Robert J. de Vos tot Nederveen Cappel • Jan Willem F. Elte • Manuel Castro Cabezas • \\ Guido H. H. Mannaerts
}

Published online: 9 August 2011

(C) Springer Science+Business Media, LLC 2011

\begin{abstract}
Background Laparoscopic sleeve gastrectomy (LSG) is becoming increasingly popular as a stand-alone procedure for the treatment of morbidly obese patients. A direct posterior approach to the angle of His was developed at our department to improve visualization of the difficult dissection of the short gastric vessels and to facilitate proper mobilization of the stomach around the left crus enabling safe realization of a tight sleeve. The technique and its preliminary results are described.

Methods LSG by posterior approach was performed in a consecutive series of 445 (110 male/335 female, age 1863 years, mean body mass index $46 \mathrm{~kg} / \mathrm{m}^{2}$ (range 35-76)) patients between 2007 and 2010.

Results Weight loss defined as mean percent excess weight loss $(\%$ EWL) was $71 \%( \pm 26 \%)$ at 1 year, $69 \%( \pm 25 \%)$ at 2 years, and $55 \%( \pm 27 \%)$ at 3 years. Sixteen patients (4\%) developed postoperative intra-abdominal hematoma, 8 patients (2\%) anastomotic leakage, and 6 patients intraabdominal abscess (1\%), requiring reoperation in 20 patients $(4 \%)$. Five patients $(1 \%)$ had pulmonary embolism. Thirty-day mortality rate was $0.2 \%$.

Conclusions LSG by the posterior approach is a safe and effective procedure, enabling a tight sleeve formation leading to satisfactory \%EWL results. Since long-term results of LSG are unknown, further studies are needed to
\end{abstract}

R. P. M. Gadiot • L. U. Biter · H. J. F. Zengerink •

R. J. de Vos tot Nederveen Cappel - G. H. H. Mannaerts ( $\square)$

Department of Surgery, Sint Franciscus Gasthuis,

Kleiweg 500,

3045 PM, Rotterdam, the Netherlands

e-mail: g.mannaerts@sfg.nl

J. W. F. Elte $\cdot$ M. Castro Cabezas

Department of Internal Medicine, Sint Franciscus Gasthuis,

Rotterdam, the Netherlands define the exact place of the LSG as a stand-alone bariatric procedure.

Keywords Laparoscopic sleeve gastrectomy · Posterior approach $\cdot$ Morbid obesity $\cdot$ Surgery

\section{Introduction}

Laparoscopic sleeve gastrectomy (LSG) is accepted as a first step bariatric procedure in severe morbidly obese (body mass index (BMI) $>50 \mathrm{~kg} / \mathrm{m}^{2}$ ) patients with high surgical and anesthesiologic risks [1-3]. The advantages of LSG are the relative simplicity, satisfying weight loss results, the lowering of ghrelin levels reducing hunger, preservation of the normal gastro-intestinal tract without foreign material, and the absence of gastric strictures allowing normal dietary options [4-6]. As a result, LSG is currently gaining popularity as a stand-alone stage bariatric procedure [7].

From a technical point of view, two LSG techniques have been described, being the "lateral-to-medial" approach, whereby stapling is performed after full mobilization of the greater curvature [8] and the reverse "medial-tolateral" approach where stapling of the complete sleeve is performed as soon as the lesser sac is entered followed by full mobilization of the greater curvature [9]. The most demanding step in this procedure is the dissection of the short gastric vessels at the angle of His where visualization can be hampered due to interposition of the stomach or omentum. Another issue is the fact that all hemorrhage accumulates in this area leading to poor visualization. This can result in esophageal injury, a less tight sleeve formation due to leaving redundant fundus, or bleeding in the splenic region. To improve visualization at the angle of His, a 
posterior approach has been developed in our department. Moreover, this approach facilitates proper dissection of the gastro-pancreatic adhesions at the left crus which is essential to create a tight sleeve. The primary aim of this article is to describe the posterior approach and its preliminary results.

\section{Materials and Method}

Between January 2007 and December 2010, 445 consecutive patients all fulfilling the criteria of the NIH guidelines [10], e.g., $18-63$ years of age and $\mathrm{BMI} \geq 40 \mathrm{~kg} / \mathrm{m}^{2}$ or $\geq 35 \mathrm{~kg} / \mathrm{m}^{2}$ with co-morbidities, underwent primary LSG using dorsal approach in the Sint Franciscus Gasthuis after an intensive multidisciplinary work-up $[11,12]$. Included patients were $335(75 \%)$ women and $110(25 \%)$ men, aged 18 to 63 years (median age 42 years). Exclusion criteria for LSG were severe complaints of gastro-esophageal reflux and extreme sweet eating. Mean pre-operative BMI was $46 \mathrm{~kg} / \mathrm{m}^{2}$ (range 35-76). Patient characteristics are described in Table 1.

\section{Statistics}

Data were analyzed using the SPSS v. 17.0 statistical program. Differences between subgroups where analyzed using MannWhitney $U$ test. The amount of weight loss was expressed as percentage excess weight loss (\%EWL) and calculated with the formula $\% \mathrm{EWL}=($ pre - operative weight - current weight $) /($ pre - operative weight - ideal weight $) \times 100 \%$ [13]. For this formula, a BMI of $25 \mathrm{~kg} / \mathrm{m}^{2}$ was taken as the upper limit of normal, i.e., our ideal weight.

\section{Surgical Technique}

The patient is positioned in reverse Trendelenburg in a socalled French position (i.e., split leg position) with both

Table 1 Patient characteristics

\begin{tabular}{ll}
\hline Patient characteristics & Total $n=445$ \\
\hline Gender, f/m & $335 / 110$ \\
Age, mean (range) & $42(18-63)$ \\
BMI kg/m ${ }^{2}$, mean & $46( \pm 6 \mathrm{SD})$ \\
BMI $>50$ & $114(26 \%)$ \\
Co-morbidity & \\
None & $190(43 \%)$ \\
Type 2 diabetes & $123(28 \%)$ \\
Obstructive sleep apnea syndrome & $28(6 \%)$ \\
Hypertension & $162(36 \%)$ \\
Hypercholesterolemia & $88(20 \%)$ \\
Artrosis & $44(10 \%)$ \\
\hline
\end{tabular}

arms placed in abduction. Elastic and intermittent pneumatic compressing stockings are applied. The surgeon stands between the patient's legs, the assistant to the patient's left, and the cameraman to the patient's right.

Phase 0-Trocar Placement Pneumoperitoneum is created by $\mathrm{CO}_{2}$ insufflation, set at $20 \mathrm{mmHg}$, through a Verress needle placed in the left mid-clavicle subcostal region. A five-port technique is employed: trocar no. $1(10-12 \mathrm{~mm})$ is placed $10-15 \mathrm{~cm}$ below the xiphoid process slightly left to the patient's mid-line ( $30^{\circ}$ angle scope); trocar no. 2 (10$12 \mathrm{~mm}$ ) is placed at the location of the Verress needle in the left upper quadrant (surgeon's right hand); trocar no. 3 (10$12 \mathrm{~mm}$ ) is placed in the right upper quadrant through the hepatic ligament (surgeon's left hand); trocar no. 4 (5 mm) is placed high epigastric in the mid-line (flexible liver retractor); and trocar no. $5(5 \mathrm{~mm})$ is placed in the lateral left abdomen (assistant's 5-mm Babcock) (Fig. 1).

Phase 1-Mobilization of the Greater Curvature A 5-mm flexible triangular liver retractor is positioned. The omentum is separated from the greater curvature using a harmonic scalpel, starting at a location halfway of the greater curvature, as it is easier to enter the omental bursa at this area. Possible slight damage of the stomach is not important at this level as this part is to be resected (Fig. 2). Further mobilization of the greater curvature is easier with less chance of bleeding when mobilization is first executed

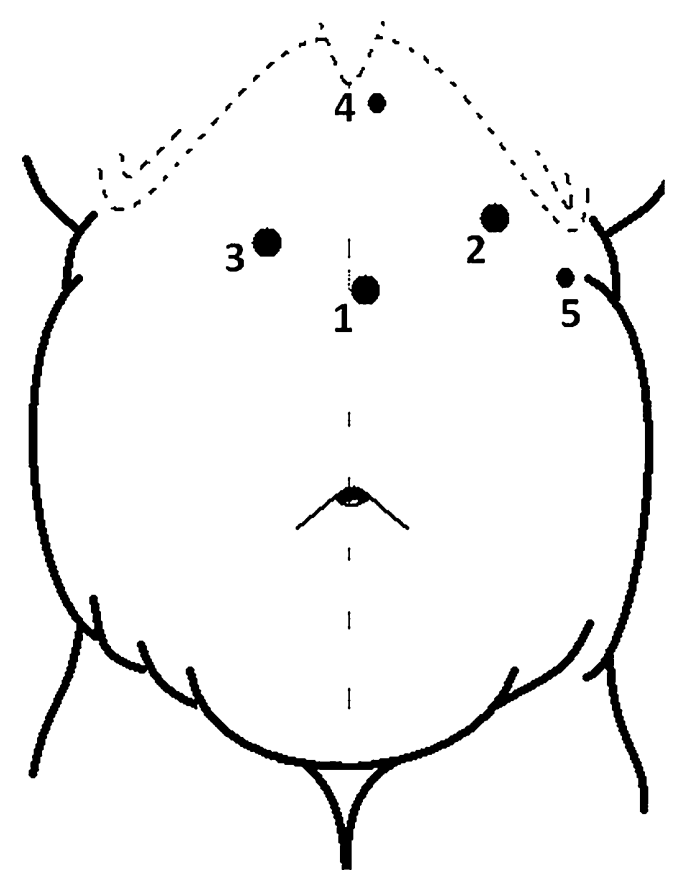

Fig. 1 Schematic view of trocar placement. 1 Optical trocar (left to the mid-line); 2 operating trocar, surgeon's right hand; 3 operating trocar, surgeon's left hand; 4 liver retractor trocar; 5 assistant's stomach trocar 


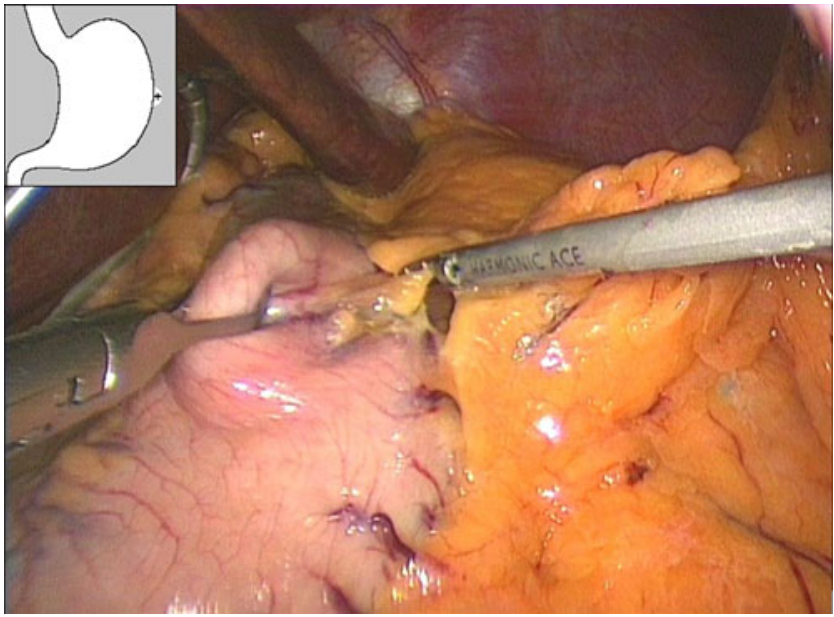

Fig. 2 Laparoscopic view of the starting point of dissection of the omentum from the greater curvature of the stomach, thereby entering the omental bursa

in an upward direction up to a level just below the spleen, leaving the small gastric arteries intact (Fig. 3). Next, the greater curvature is dissected downward to the point where the stomach is attached to the pancreas $(2 \mathrm{~cm}$ proximal to the pylorus) (Fig. 4).

Phase 2-Posterior Mobilization of the Stomach and Angle of His The assistant grabs the stomach with a 5-mm Babcock grasper high at the dorso-lateral fundus (no. 5 trocar) and pulls it to the patient's left as well as in ventral direction. Next, a 10-mm Babcock grasper (no. 3 trocar) is placed posterior to the stomach pushing it in the patient's right and ventral direction thus creating a tented shaped configuration of the stomach (Fig. 5). The gastro-pancreatic adhesions are carefully dissected in the gastro-pancreatic surgical plane, avoiding damage to the branches of the left gastric artery, in cranial direction towards the angle of His.

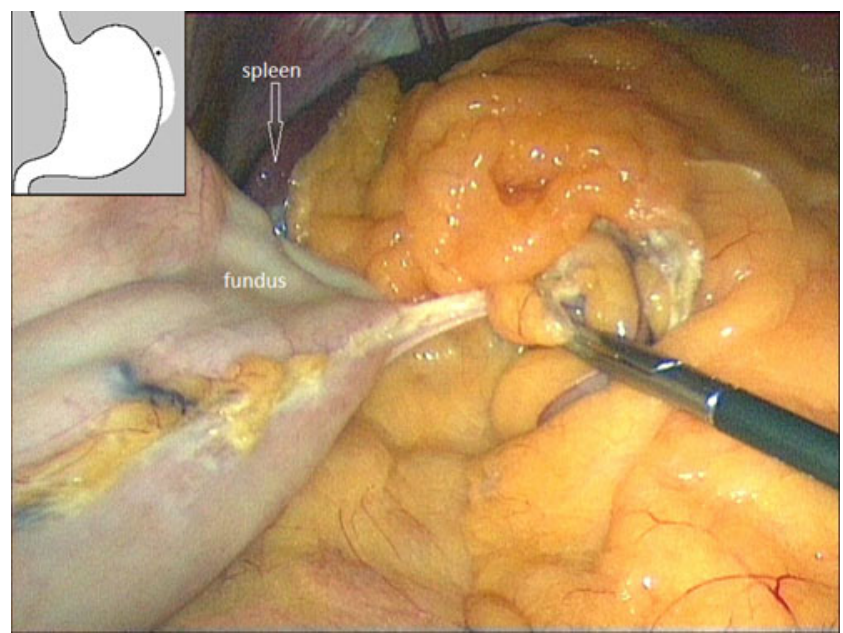

Fig. 3 Laparoscopic view of the mobilization of the greater curvature in cranial direction to a level just below the spleen

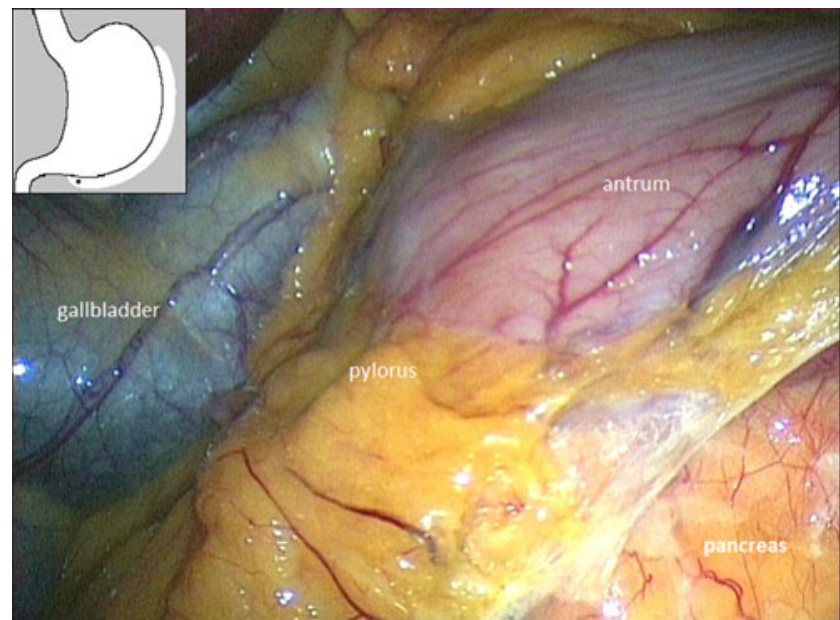

Fig. 4 Laparoscopic view of the dissection in caudal direction up to the level where the greater curve is attached to the head of the pancreas (approximately $2-3 \mathrm{~cm}$ to the left of the pylorus)

At this point, the dorsal part of the spleen can be visualized, and the short gastric vessels at the angle of His are dissected. Then, the $10-\mathrm{mm}$ Babcock is placed dorsal to the stomach at the angle of His, pushing the stomach to the patient's right and ventrally, followed by slight retraction of this Babcock. This maneuver will further visualize the left diaphragm crus and the space between the crus and spleen (Fig. 6). Proper prior posterior gastric mobilization completely exposing the left crus is important to enable easy stapling without harming the spleen and without leaving redundant fundus behind. The crural branch of the left phrenic vein situated on the left crus in parallel direction (Fig. 7) can be used as a landmark by dissecting lateral to this vascular structure in order not to harm the esophagus when dissecting the angle of His. This applies for the dorsal as well as the ventral phase (see phase 3). At this point, the most difficult and dangerous part of the operation is

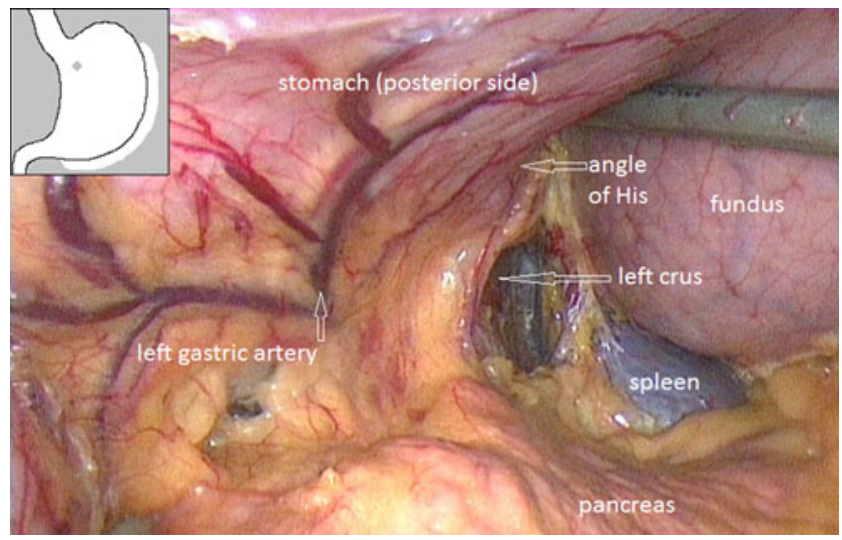

Fig. 5 Laparoscopic view of the gastro-pancreatic adhesions posterior to the stomach. A tented shaped configuration of the stomach is created by lifting the stomach to ventrolateral by the assistant's 5-mm Babcock. The gastro-pancreatic adhesions are released in upward direction towards the angle of His 

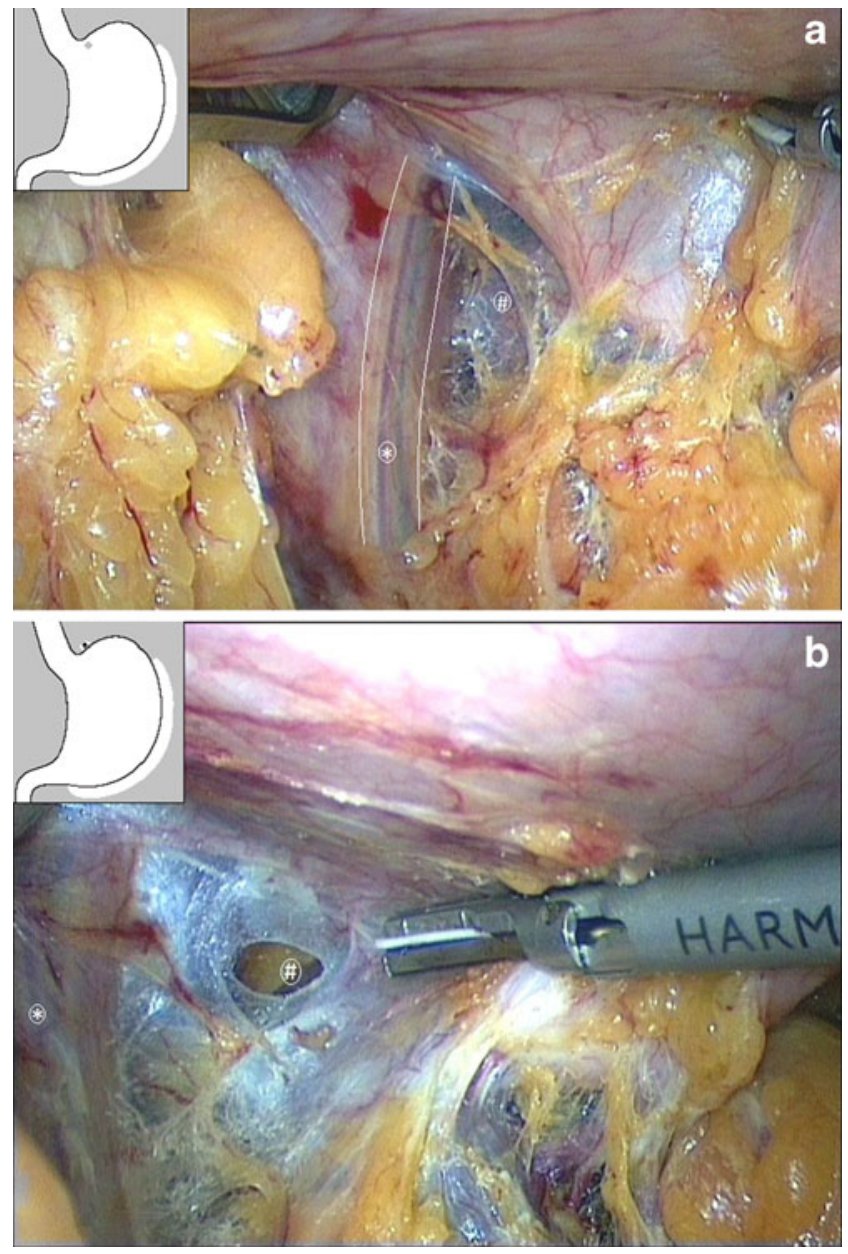

Fig. 6 a, b Laparoscopic view of the dorsal mobilization of the angle of His, thus visualizing the left diaphragm crus and the space between the crus and the spleen. Asterisks crural branch of left phrenic vein. Number signs spleen

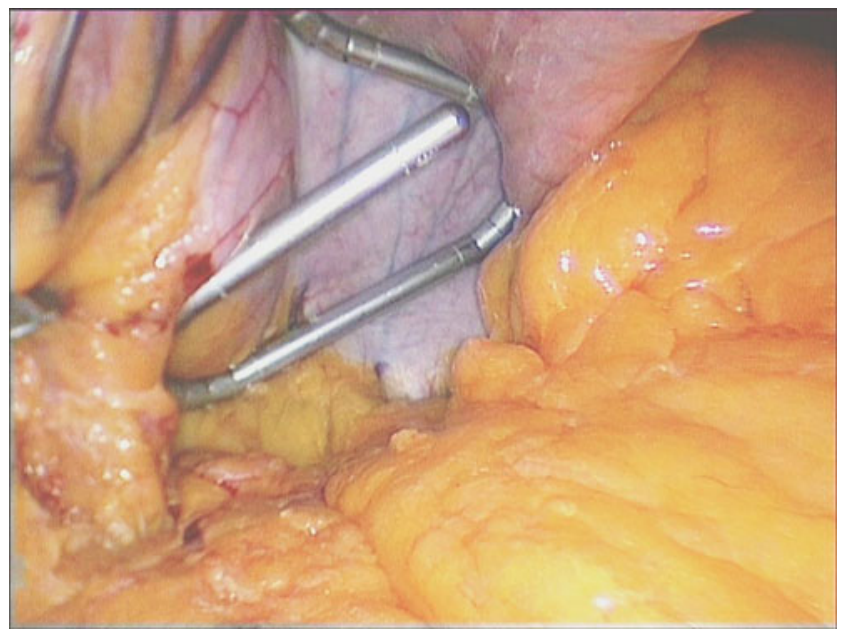

Fig. 7 Alternatively, the liver retractor (flexible triangular retractor) can be placed behind the stomach in superobese patients to improve visualization of dorsal gastric dissection completed. Alternatively, in superobese patients where visualization of the dorsal gastric mobilization can be bothered, the liver retractor can be placed in the bursa omentalis behind the stomach in order to push the stomach in ventral direction, thus creating visualization (Fig. 7).

Phase 3-Ventral Mobilization of Angle of His Next, the ventral aspect of the angle of His is mobilized by dissecting the peritoneal fold above the Belsy's fat (at the cardioesophageal junction) (Fig. 8). Note that the neurovascular bundle, mentioned in the previous phase, is easily identified on the ventral side of the stomach and can be used as a landmark.

Phase 4-Finalizing Mobilization of the Greater Curvature by Dissecting the Remaining Small Gastric Vessels Mobilization of the greater curvature is finished by dissection of the small gastric vessels in cranial direction along the greater curvature (Fig. 9). Now that the greater curvature is completely mobilized, the last remaining adhesions of the

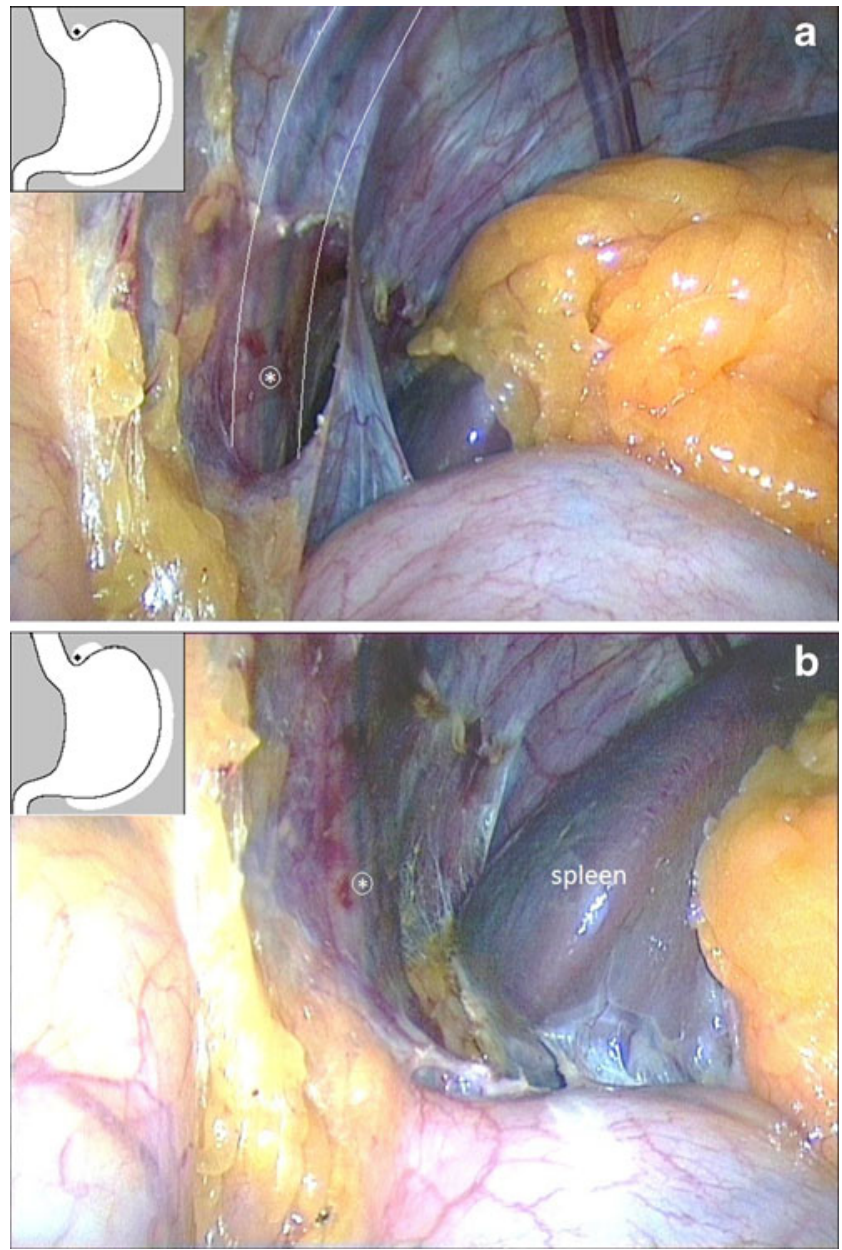

Fig. 8 a, b Laparoscopic view of the dissection of the ventral aspect of the angle of His at the cardio-esophageal junction (above the Belsy's fat). Asterisks crural branch of left phrenic vein 




Fig. 9 Laparoscopic view of the dissection of the greater curve, i.e., small gastrics, at the spleen level in order to complete the mobilization of the greater curve

stomach at the diaphragmatic crus are released gently in order not to damage the esophagus (Fig. 10).

Phase 5-Stapling All staplers $(60 \mathrm{~mm} \times 3.5 \mathrm{~mm}$ linear cutter Echelon, Ethicon, using green cartridge $4.1 \mathrm{~mm}$ for men, gold cartridge $3.8 \mathrm{~mm}$ for women) are fired using the surgeon's left hand through the patient's right no. 3 trocar, as stapling through the contralateral (trocar no. 2) increases the risk of stapling into the calibration tube. First stapler is fired at a point $2-3 \mathrm{~cm}$ proximal to the pylorus. Before stapling, the assistant's 5-mm Babcock (no. 5 trocar) holds the distal stomach at the greater curvature just proximal to the left side of the stapler keeping the stapler in place. Meanwhile, the surgeon uses the 10-mm Babcock with his right hand to pull the lesser curvature out of the tip of the stapler in order to make sure that the stomach is not stapled too narrow at the lesser curve (Fig. 11). After firing the first stapler, a 34F gastric calibration tube is inserted and guided over the dorsal side of the stomach along the first stapler line up to the pylorus (Fig. 12). Since the distal stomach has been stapled once, the tube will stay in place in the lesser curvature. Next, another four to five staplers are fired to finish the gastric sleeve. Before stapling, the assistant's 5$\mathrm{mm}$ Babcock grabs the stomach repeatedly at its greater curvature at a location where the lateral curvature makes a vault and pulls it to the patient's caudal and left side. At the same time, the surgeon pulls the already stapled greater curve of the stomach caudally with a 10-mm Babcock (no. 2 trocar) while he places the stapler tight to the tube (Fig. 13). Before firing the stapler, proper dorsal placement is checked to avoid leaving too much dorsal stomach volume, which would result in a too wide sleeve. Furthermore, the $34 \mathrm{~F}$ tube is moved slightly by the anesthesiologist under laparoscopic view, focusing on the
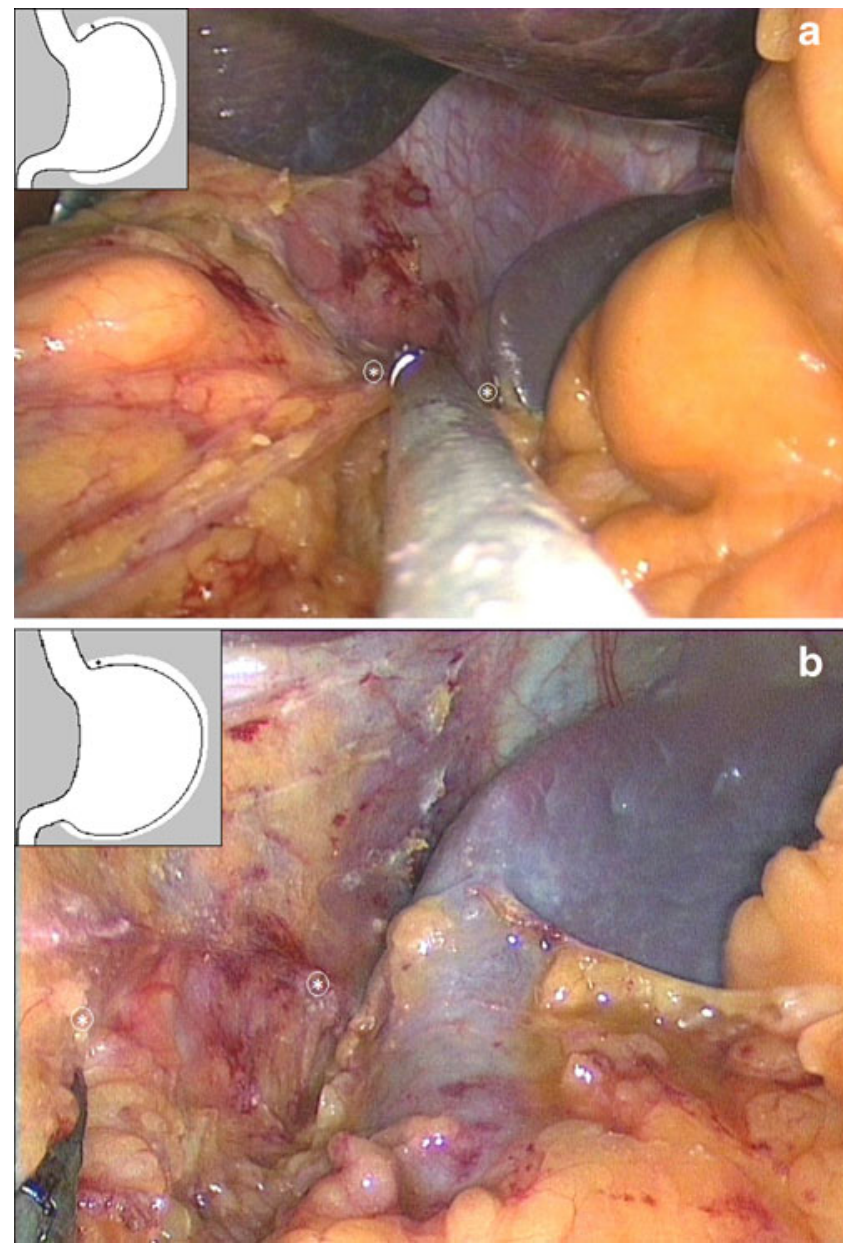

Fig. 10 a, b Laparoscopic view of the completely mobilized angle of His, where the last remaining adhesions (asterisks) of the stomach at the left diaphragmal crus are released very gently in order not to damage the esophagus, after which the angle of His can be fully stretched (b)

tip of the $34 \mathrm{~F}$ tube, assuring that this tube is not caught within the stapler. After having fired four to five staplers, the last stapler is fired less tight to the tube leaving Belsy's fat at the gastric sleeve. This avoids stapling into the esophagus and thereby reduces the chance of anastomotic leakage (Fig. 14). Staple line bleeding is controlled by the harmonic scalpel and when necessary with clips.

Phase 6-Removal of Gastric Specimen The specimen is removed through the patient's left trocar (no. 2) which is dilated digitally. Next, the trocar opening is closed with a suture passed through the fascia by a clamp (Pean clamp), and local anesthesia (Rapifen) is injected in the fascia at this location. The gastric $34 \mathrm{~F}$ tube is now removed under laparoscopic view by the anesthesiologist without using suction on the tube in the gastric area until it is in the esophagus (Fig. 15). In case of bleeding, stapling problems, or difficult realization of the gastric sleeve, a drain is placed 


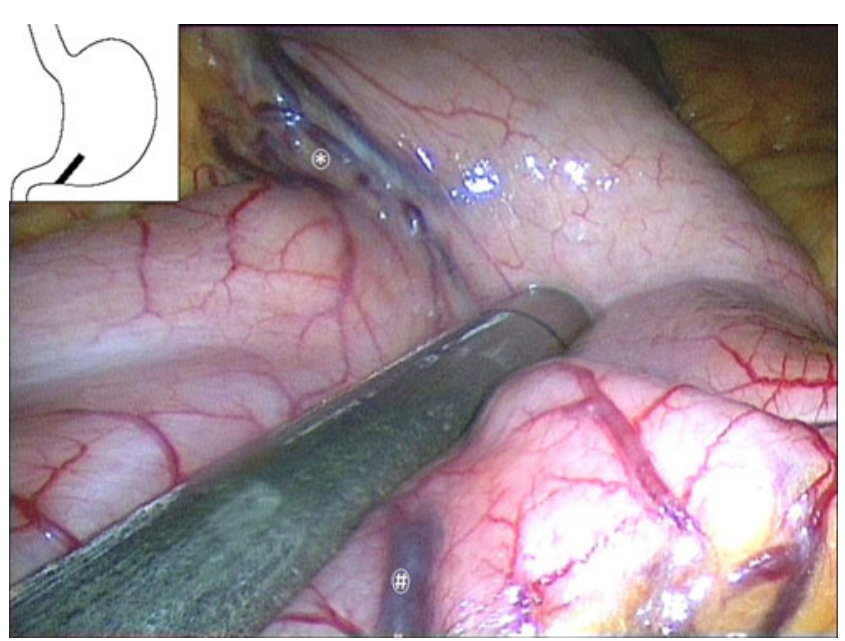

Fig. 11 Laparoscopic view of the placement of the first stapler by the surgeon's left hand through the patient's right trocar at a location 2$4 \mathrm{~cm}$ proximal to the pylorus. The assistant's 5-mm Babcock (number sign) holds the stomach at the greater curvature just proximal to the left side of the stapler keeping the stapler in place while the surgeon uses the 10-mm Babcock with his right hand to pull the lesser curvature (at crows foot (asterisk)) out of the tip of the stapler in order to make sure that the distal stomach is not stapled to narrow

along the sleeve gastrectomy through the patient's right trocar (no. 3).

Postoperative Policy Oral crystal liquids are allowed at the day of surgery. The next day, only fluids are admitted for 2 weeks. At the first or second postoperative day, the patient is discharged. CT scan with oral diluted contrast is performed in patients with early signs of infection (HR $>120 / \mathrm{min}$, (sub)febrile temperature and elevated CRP), but in case of high suspicion of leakage, a diagnostic laparoscopy is performed. Gastrografin study and/or gas-

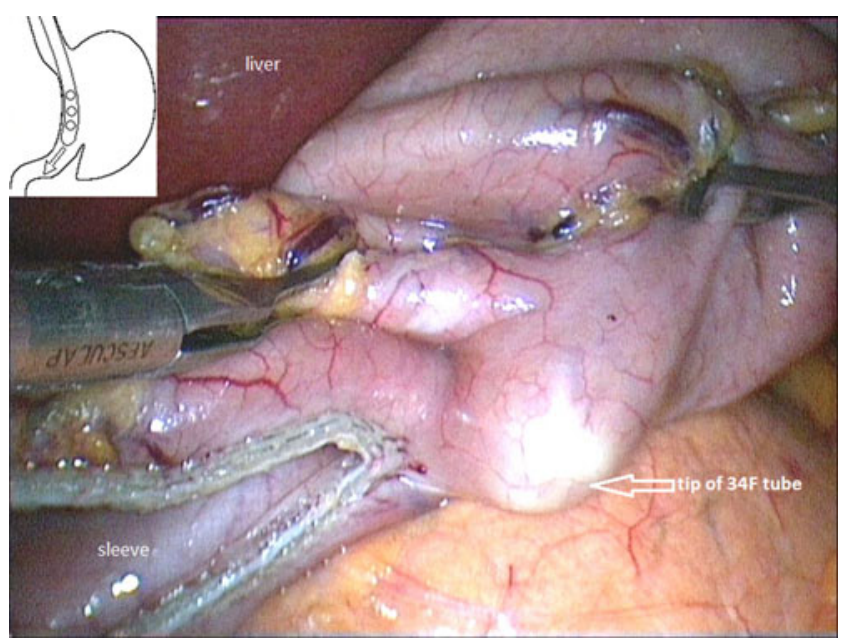

Fig. 12 Schematic view of the insertion of the 34F gastric tube under assistance of two 10-mm Babcocks guided over the dorsal side of the stomach along the first stapler line and passed up to the pylorus

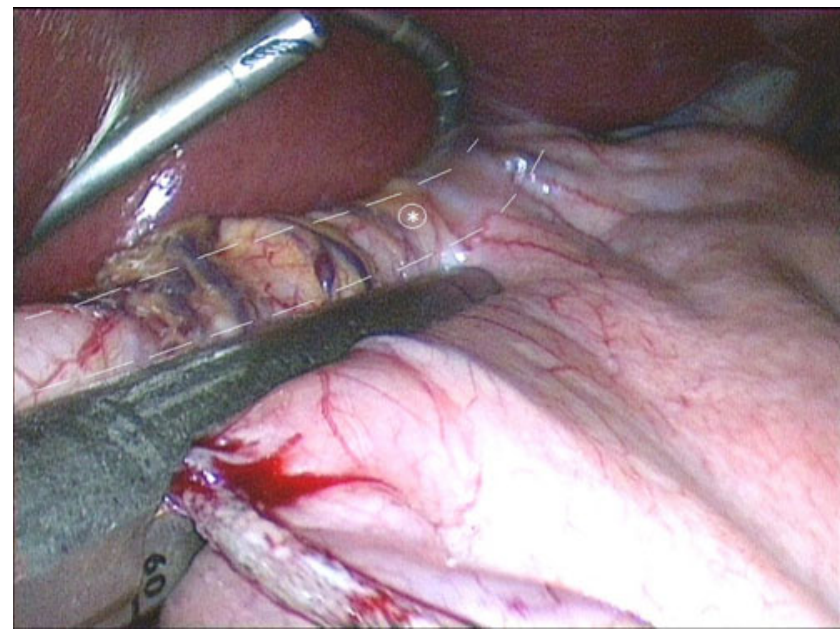

Fig. 13 Laparoscopic view of the creation of a tight sleeve by placement of the stapler tight to the sleeve and pulling the greater curvature to the patient's left side using the assistant's 5-mm Babcock (no. 5 trocar) placed at a higher level and the surgeon's 10-mm Babcock (no. 2 trocar) placed at the already stapled greater curve of the stomach. Asterisk position of the 34F gastric tube

troscopy is performed in case of severe dysphagia or mild dysphagia lasting more than 3 months postoperatively. Furthermore, elastic compression stockings (for the duration of 2 weeks postoperatively) and pneumatic dynamic leg compression sleeves (for $24 \mathrm{~h}$ postoperatively) are used, and subcutaneous low molecular weight heparin (LMWH) injection (daily dose of $5,000 \mathrm{U}$ ) is administered until discharge. As a result of a relatively high rate of pulmonary embolism at the beginning of this study, LMWH is currently prescribed up until 2 weeks postoperatively. In addition, a proton pump inhibitor (esomeprazole $40 \mathrm{mg}$ ) is

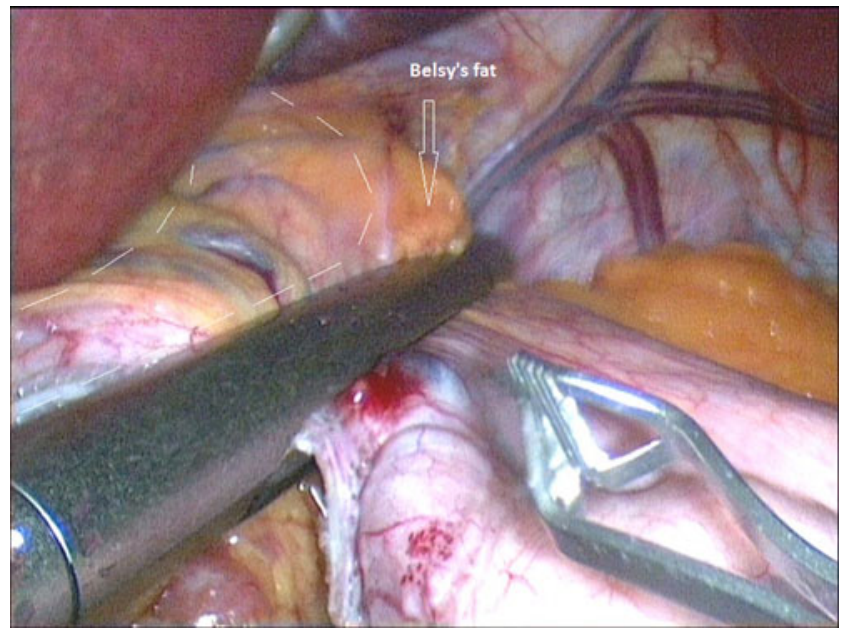

Fig. 14 Laparoscopic view of the placement (less tight) of the last stapler at the angle of His, leaving Belsy's fat (asterisk) at the gastric sleeve, in order to avoid stapling into the esophagus and thereby reducing the chance of anastomotic leakage 


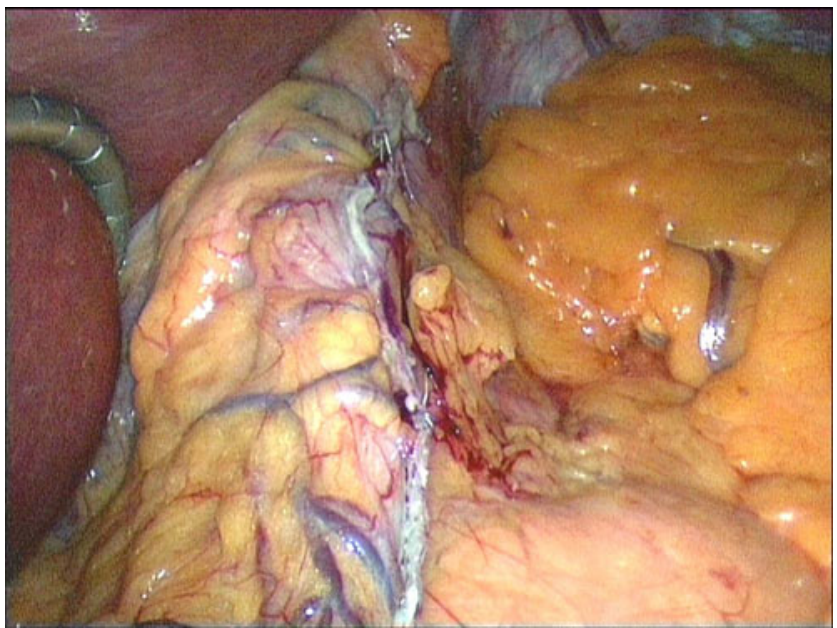

Fig. 15 Laparoscopic view of the final situation after removal of the $34 \mathrm{~F}$ gastric tube. There is no redundant gastric fundus at the proximal sleeve

applied for 6 weeks to prevent marginal ulcer formation. Outpatient clinic visits are scheduled at 1, 4, and 12 months and then every year.

\section{Results}

The mean operating time of the dorsal LSG was 41 min (range 19-196). Intra-operative complications were stapling of the nasogastric tube in two patients $(0.4 \%)$ of which none required conversion into a laparoscopic gastric bypass. Operative and postoperative complications are listed in Table 2.

Sixteen patients $(4 \%)$ developed postoperative intraabdominal hematoma, eight patients $(2 \%)$ anastomotic leakage, six patients intra-abdominal abscess (1.3\%), and five patients $(1.1 \%)$ had pulmonary embolism. Reoperation for complications was necessary in 20 patients $(4.5 \%)$ for leakage $(n=4)$, hematoma $(n=12)$, and intra-abdominal abscess $(n=4)$. The mean postoperative hospital stay was 2 days (range 1-61). Two patients died (0.4\%), one as result of pulmonary embolism and one as a result of a conversion operation to gastric bypass for a gastro-cutaneous fistula 9 months after the LSG.

The mean weight change was $41 \mathrm{~kg}( \pm 15 \mathrm{~kg})$ at 1 year $(n=$ $272), 40 \mathrm{~kg}( \pm 18 \mathrm{~kg})$ at 2 years $(n=105)$, and $33 \mathrm{~kg}( \pm 21 \mathrm{~kg})$ at 3 years $(n=40)$. The mean excess weight loss was $71 \pm$ $26 \%$ (range $-7-145$ ) at 1 year, $69 \pm 28 \%$ (range $-11-125)$ at 2 years, and $55 \pm 27 \%$ (range $-14-107$ ) at 3 years. The reduction in mean BMI was 14,14 , and $12 \mathrm{~kg} / \mathrm{m}^{2}$, respectively.

Subgroup analysis was performed to compare \%EWL outcome in patients with pre-operative BMI of $\leq 50 \mathrm{~kg} / \mathrm{m}^{2}$
Table 2 Operation characteristics

\begin{tabular}{|c|c|}
\hline Operation characteristics & $\begin{array}{l}\text { Total } n= \\
445\end{array}$ \\
\hline Conversion to open procedure & 0 \\
\hline \multicolumn{2}{|l|}{ Peri-operative complications } \\
\hline Minor blood loss & $4(0.9 \%)$ \\
\hline Stapling of 34F gastric tube (corrected laparoscopically) & $2(0.4 \%)$ \\
\hline Minor liver laceration & $3(0.7 \%)$ \\
\hline Minor spleen laceration & $3(0.7 \%)$ \\
\hline \multicolumn{2}{|l|}{ Complications } \\
\hline \multicolumn{2}{|l|}{ Postoperative complications (in hospital) } \\
\hline Intra-abdominal hematoma & $7(1.6 \%)$ \\
\hline Leakage & $5(1.1 \%)$ \\
\hline Pneumonia & $3(0.7 \%)$ \\
\hline Hematoma of abdominal wall & $3(0.7 \%)$ \\
\hline Reoperation for complications & $8(1.8 \%)$ \\
\hline Gastric stenting for leakage & $5(1.1 \%)$ \\
\hline Mortality (due to pulmonary embolism) & $1(0.2 \%)$ \\
\hline \multicolumn{2}{|l|}{ Postoperative complications ( $<6$ weeks after discharge) } \\
\hline Intra-abdominal hematoma & $9(2.0 \%)$ \\
\hline Leakage & $3(0.7 \%)$ \\
\hline $\begin{array}{l}\text { Pulmonary embolism }(16,19,25 \text {, and } 75 \text { days } \\
\text { postoperatively) }\end{array}$ & $4(0.9 \%)$ \\
\hline Sustaining dysphagia & $9(2.0 \%)$ \\
\hline Symptomatic cholecystolithiasis & $10(2.2 \%)$ \\
\hline Reoperation for complications & $12(2.7 \%)$ \\
\hline Intra-abdominal abscess & $6(1.3 \%)$ \\
\hline Mortality & $1(0.2 \%)$ \\
\hline
\end{tabular}

to patients with pre-operative BMI of $\geq 50 \mathrm{~kg} / \mathrm{m}^{2}$, which showed a significant difference in favor of patients with a pre-operative BMI of $<50$ at 1 and 2 years of follow-up $(p<0.001$ and $p=0.04$, respectively). Patients with a preoperative BMI of $\leq 60 \mathrm{~kg} / \mathrm{m}^{2}$ also showed significant ( $p<$ 0.001 ) better results in \%EWL after 1-year follow-up in comparison to patients with a pre-operative BMI of $\geq 60 \mathrm{~kg} / \mathrm{m}^{2}$ (Table 3 ).

Conversion into a laparoscopic Roux-en-Y gastric bypass (LRYGB) in a later phase (Fig. 16) was necessary in 16 patients $(3.6 \%)$, for persisting dysphagia in $2(0.5 \%)$, and for unsatisfactory weight loss in 14 (3.1\%).

After a minimal follow-up period of 1 year (thus excluding the patients receiving LSG in 2010), type 2 diabetes was cured in 40 patients $(47 \%)$, improved (reduced medication) in 15 patients (18\%), and unchanged in 30 patients (35\%). After 1 year of follow-up, significantly more patients with a high $\% \mathrm{EWL}(>55 \%)$ were cured of diabetes $(n=26(60 \%)$ vs. $n=14(33 \%), p=0.012)$. However, this significant difference did not sustain at 2 and 3 years of follow-up. 
Table $3 \% \mathrm{EWL}$ outcome comparison between pre-operative $\mathrm{BMI}<50$ and $\mathrm{BMI}>50$ and pre-operative $\mathrm{BMI}<60$ and $\mathrm{BMI}>60$

\begin{tabular}{lllc}
\hline & BMI & & \\
\hline \%EWL 1 year, mean (SEM) & $76 \%(1.9), n=196^{\mathrm{a}}$ & $56 \%(2.4), n=76^{\mathrm{b}}$ & $<0.001$ \\
\%EWL 2 years, mean (SEM) & $71 \%(3.0), n=74^{\mathrm{a}}$ & $61 \%(4.0), n=31^{\mathrm{b}}$ & 0.04 \\
$\%$ EWL 3 years, mean (SEM) & $55 \%(5.7), n=25^{\mathrm{a}}$ & $56 \%(7.5), n=15^{\mathrm{b}}$ & 0.7 \\
\%EWL 1 year, mean (SEM) & $72 \%(1.7), n=244^{\mathrm{c}}$ & $48 \%(3.8), n=28^{\mathrm{d}}$ & $<0.001$ \\
$\%$ EWL 2 years, mean (SEM) & $69 \%(2.6), n=100^{\mathrm{c}}$ & $58 \%(5.1), n=5^{\mathrm{d}}$ & 0.2 \\
\%EWL 3 years, mean (SEM) & $55 \%(4.7), n=39^{\mathrm{c}}$ & $62 \%, n=1^{\mathrm{d}}$ & 0.7 \\
\hline
\end{tabular}

SEM standard error of mean

${ }^{\mathrm{a}} \mathrm{BMI}<50$

${ }^{\mathrm{b}} \mathrm{BMI}>50$

${ }^{\mathrm{c}} \mathrm{BMI}<60$

${ }^{\mathrm{d}} \mathrm{BMI}>60$

\section{Discussion}

LSG is accepted as the first step bariatric procedure (followed by either duodenal switch or Roux-en-Y gastric bypass) in the treatment of superobese patients or in patients with high operative risk [3]. Currently, LSG is becoming increasingly popular as an isolated operation, because it represents a relatively straightforward bariatric procedure, resulting in satisfying weight loss, reduced perception of hunger, preservation of normal gastro-intestinal tract without foreign material, and the absence of gastric strictures allowing normal dietary habits without symptoms of dumping [4-6]. These advantages of LSG and its satisfying weight loss in the step-up procedure have promoted the use of LSG as a stand-alone bariatric procedure, resulting in a \%EWL ranging from $47 \%$ to $83 \%$ (1 year), $47-61 \%$ (2 years), 60 $66 \%$ ( 3 years), $58 \%$ ( 4 years) and 55\% (5 years) [2, 14-19],

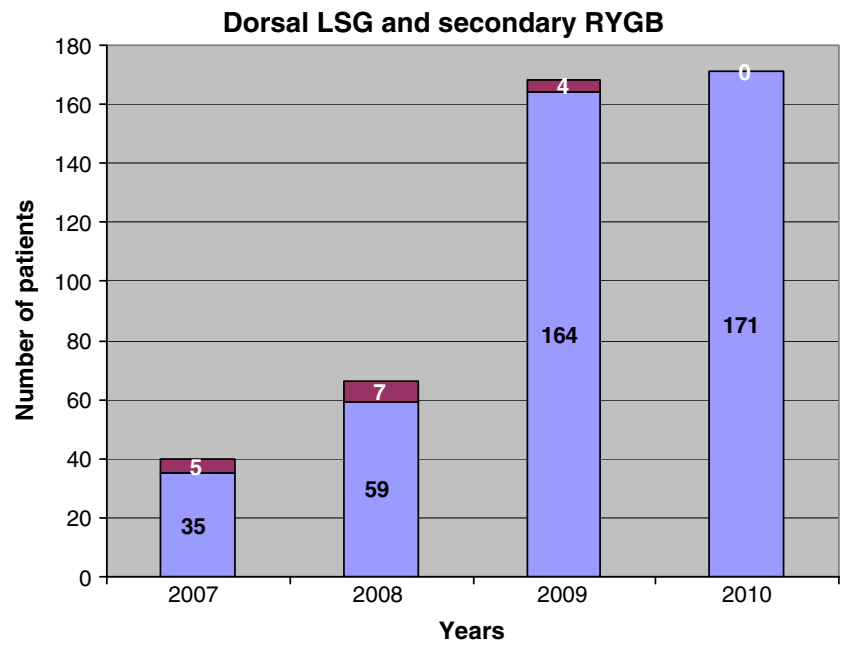

Fig. 16 Number of LSG procedures per year with number of patients receiving second step LRYGB after follow-up. Second step LRYGB numbers in white which is comparable with the $\%$ EWL of $71 \%$ at 1 year, $69 \%$ at 2 years, and $55 \%$ at 3 years in this study. Small prospective series show significant better short-term weight loss results of LSG in comparison with LRYGB (\%EWL $70 \%$ vs. $60 \%$ at 1 year) as a result of markedly and consistently reduced ghrelin levels in addition to increased PYY levels after LSG [20] and comparable improvement in the metabolic control of diabetes [21, 22]. Nevertheless, long-term weight loss of this relative new procedure is not clear as gastric capacity might increase in time.

Realization of a small gastric sleeve seems to be important as smaller bougie sizes affect weight loss results and have (according to the LaPlace law) less chance to dilate [23]. In order to realize a tight sleeve and to avoid damage to the esophagus and the spleen at the angle of His, three technical aspects are essential: (1) complete (especially dorsal) liberation of the gastric fundus (to avoid redundant fundus), (2) proper traction on the dorsal layer of the stomach when placing the linear stapler (to avoid realization of a wide gastric sleeve), and (3) firing of the final linear stapler slightly lateral to the angle of His (to avoid encroachment of the esophagus). In bariatric literature, two different approaches have been described. Gagner et al. described the lateral-to-medial approach (mobilization of greater curve followed by stapling) [8], which has the advantage of optimal calibration of the tight sleeve, but often the disadvantage of poor visualization of the dissection of the small gastric vessels at the medial site of the spleen due to the fact that the stomach and omentum are blocking the view. Moreover, dissection of the gastrosplenic ligament can result in bleeding. Hematoma formation at this dangerous point near the angle of His increases the risk of damage to the spleen, esophagus, or stomach when dissecting in that area or when the stapler is placed. Himpens et al. described the medial-to-lateral approach (stapling performed as soon as the lesser sac is entered followed by mobilization of greater curve) [9]. This has the 
advantage of easier dissection of the small gastric vessels but the disadvantage of poorer control of achieving a tight sleeve as it is more difficult to pull the dorsal layer of the stomach into the stapler. We developed a posterior approach in which the angle of His is mobilized at the beginning of the procedure, directly after partial mobilization of the greater curve and dissection of the dorsal gastro-pancreatic adhesions in the cranial direction. This approach, in which the stomach is tented ventrally, has the advantage of better visualization when dissecting the dorsal angle of His and the small gastric vessels, thus minimizing the chance of harming the esophagus, stomach, or spleen. The advantage of this strategy is the lack of blood loss bothering dissection and the absence of greater omentum bothering vision. As a result, proper tight calibration of the sleeve can be easily performed. Another advantage of this technique is that, due to its proper visualization and mobilization of the gastropancreatic adhesions, the left gastric artery branches are well mobilized, thus avoiding the chance of these structures being damaged within the stapler. Besides gentle dissection in the angle of His area, it is also important that the final linear stapler does not staple Belsy's fat of the gastroesophageal junction. This may result in stapling into the esophagus which results in a higher chance of leakage of the gastric sleeve at the angle of His due to high intraluminal pressure as a result of the restrictive nature of the procedure. In this large series of LSG procedures, we experienced this problem in the early years resulting in an overall $1.8 \%$ leakage. In some of these patients $(n=2)$, staples were observed in the esophagus when endoluminal stents were placed to control the leakage. Furthermore, we experienced subphrenic abscess in $0.2 \%$, hematoma in $4 \%$, and reoperation in 4\%. Apart from hematoma, these rates are comparable to the $0.2 \%, 0.4 \%$, and $3.6 \%$ rates, respectively, in the study of Frezza et al., wherein LSG results of a total of 810 patients from 15 studies were assembled [24]. The higher rate of hematomas was due to the fact that we tried to reduce the initial higher rate of pulmonary embolism by a higher LMWH dosage (60 IU/ $\mathrm{kg}$ ), which was later abandoned as we experienced more abdominal hematomas. Continuation of administration of $5,000 \mathrm{IU}$ of LMWH for a period of 2 weeks combined with introduction of dynamic pneumatic leg compression sleeves perioperatively solved these problems of hematoma and also of pulmonary embolism, even though the use of high intra-abdominal pressure $(20 \mathrm{mmHg})$ is still applied for this procedure. Although expensive, additional use of buttress material to reinforce the staple line might help further reduce staple line bleeding $[25,26]$. However, the use of the harmonic scalpel is satisfactory in most cases to control staple line bleeding. Clips are not routinely used for this matter as they might cause stapling failure if a gastric bypass would be required in the future.
Overall mortality rate in this series of $0.4 \%$ is also comparable with the $0.5 \%$ in the study of Frezza et al. [24]. Strictures did not occur in this study as opposed to $0.7 \%$ in the study of Frezza et al., and it is suggested to be associated with oversewing the staple line [1]. In our series, oversewing was performed at the beginning of the study and did not prevent anastomotic leakage.

There is a wide range in mean operation time in the literature for LSG ranging from 25 to $115 \mathrm{~min}[18,27]$. An experienced surgeon needs approximately $30 \mathrm{~min}$ to perform dorsal LSG in normal cases ranging to $45 \mathrm{~min}$ in more difficult superobese male patients.

In this study, a marked decline in \%EWL after 3 years of follow-up was noticed when compared to the 1- and 2-year \% EWL results. This could be explained by the surgeon's learning curve and the relatively small group of patients with a follow-up of 3 years, as well as the fact that we became more strict on performing LRYGB for sweet eaters over the last 2 years. However, possible dilatation of the sleeve over the years and possible change in dietary habits (i.e., more sweet eating) can also be an explanation for this weight regain.

Patients with a high pre-operative BMI had a significantly lower \%EWL outcome after 1 and 2 years of followup which is consistent with the results stated in the literature $[23,28]$. Due to the fact that medical costs become more important, attempts for approval and reimbursement for a staged approach in superobese patients is likely to be met with great resistance. Especially, as with increased experience and improvements in technique, an argument can be made for proceeding directly with a LRYGB as overall morbidity and mortality are comparable with a staged approach even in patients with BMI $>60 \mathrm{~kg} / \mathrm{m}^{2}[13$, 29]. On the other hand, LRYGB is associated with the discomfort of dumping syndrome and, in some cases, weight regain or insufficient weight loss [30].

LSG by using the posterior approach offers safe proper visualization of dissection at the dangerous location at the angle of His and provides proper mobilization of the dorsal fundus at the left crus, which facilitates calibration of a tight gastric sleeve. Based on the satisfactory short-term results stated in literature, LSG might be a promising bariatric procedure for certain subgroups of morbidly obese patients. However, a randomized trial comparing LSG with LRYGB and/or Lap Band is necessary to determine durability and long-term merits (\%EWL, QOL, Qually's) of these subgroups of morbidly obese patients treated by LSG in comparison to these other bariatric procedures. Such a trial is currently under construction by our department.

Conflict of Interest The authors of this manuscript, R.P.M. Gadiot, L.U. Biter, J.F. Zengerink, R.J. de Vos tot Nederveen Cappel, J.W.F. Elte, M. Castro Cabezas, and G.H.H. Mannaerts, have no conflicts of interest or financial ties to disclose. 


\section{References}

1. Cottam D, Qureshi FG, Mattar SG, et al. Laparoscopic sleeve gastrectomy as an initial weight-loss procedure for high risk patients with morbid obesity. Surg Endosc. 2006;20:859-63.

2. Silecchia G, Boru C, Pecchia A, et al. Effectiveness of laparoscopic sleeve gastrectomy (first stage of biliopancreatic diversion with duodenal switch) on co-morbidities in super-obese high-risk patients. Obes Surg. 2006;16:1138-44.

3. Regan JP, Inabnet WB, Gagner M, et al. Early experience with two-stage laparoscopic Roux-en-Y gastric bypass as an alternative in the super-obese patient. Obes Surg. 2003;13:861-4.

4. Cummings DE. Ghrelin and the short-and long-term regulation of appetite and body weight. Physiol Behav. 2006;89:71-84.

5. Ariyasu H, Takaya K, Tagami T, et al. Stomach is a major source of circulating ghrelin, and feeding state determines plasma ghrelin-like immunoreactivity levels in humans. J Clin Endocrinol Metab. 2001;86:4753-8.

6. Langer FB, Reza Hoda MA, Bohdjalian A, et al. Sleeve gastrectomy and gastric banding: effects on plasma ghrelin levels. Obes Surg. 2005;15:1024-9.

7. Papailiou J, Albanopoulos K, Toutouzas KG, et al. Morbid obesity and sleeve gastrectomy: how does it work? Obes Surg. 2010;20:1448-55.

8. Moj J, Pomp A, Dakin G, et al. Laparoscopic sleeve gastrectomy for morbid obesity. Am J Surg. 2008;196:e56-9.

9. Dapri G, Vaz C, Cadiere GB, Himpens J. A prospective randomized study comparing two different techniques for laparoscopic sleeve gastrectomy. Obes Surg. 2007;17:1435-41.

10. Gastrointestinal surgery for severe obesity. National Institutes of Health Consensus Development Conference Draft Statement. Obes Surg 1991;1:257-66.

11. Elte JW, Castro Cabezas M, Mannaerts GH, et al. Proposal for a multidisciplinary approach to the patient with morbid obesity: the Sint Franciscus Hospital morbid obesity program. Eur J Intern Med. 2008;19:92-8.

12. Elte JW, Mannaerts GH, Vrijland WW, et al. Morbid obesity and bariatric surgery. Med Interna. 2008;5:65-70.

13. Cherian PT, Tentzeris V, Sigurdsson A. Variation of outcome in weight loss with band volume adjustments under clinical and radiological control following laparoscopic adjustable gastric banding. Obes Surg. 2010;20:13-8.

14. Han SM, Kim WW, Oh J. Results of laparoscopic sleeve gastrectomy at one year in morbidly obese Korean patients. Obes Surg. 2005;15:1469-75.

15. Himpens J, Dapri G, Cadiere GB. A prospective randomized study between laparoscopic gastric banding and laparoscopic isolated sleeve gastrectomy: results after 1 and 3 years. Obes Surg. 2006;16:1450-6.
16. Moon Hs, Kim WW, Oh JH. Results of laparoscopic sleeve gastrectomy at 1 year in morbidly obese Korean patients. Obes Surg. 2005; 15:1469-75.

17. Langer BF, Bohdjalian A, Felb XF, et al. Does gastric dilatation limit the success of sleeve gastrectomy? Obes Surg. 2006;16:166-71.

18. Roa EP, Kaidar-person O, Pinto D, et al. Laparoscopic sleeve gastrectomy as treatment for morbid obesity: technique and shortterm outcome. Obes Surg. 2006;16:1323-6.

19. Bohdjalian A, Langer FB, Shakeri-Leidenmuhler S, et al. Sleeve gastrectomy as sole and definitive bariatric procedure: 5 -year results for weight loss and ghrelin. Obes Surg. 2010;20:535-40.

20. Karamanakos SN, Vagenas K, Kalfarentzos F, et al. Weight loss, appetite suppression, and changes in fasting and postprandial ghrelin and peptide-YY levels after Roux-en-Y gastric bypass and sleeve gastrectomy: a prospective, double blind study. Ann Surg. 2008;247:401-7.

21. Vidal J, Ibarzabal F, Romero F, et al. Type 2 diabetes mellitus and metabolic syndrome following sleeve gastrectomy in severely obese subjects. Obes Surg. 2008;18:1077-82.

22. Peterli R, Wolnerhanssen B, Peters T, et al. Improvement in glucose metabolism after bariatric surgery: comparison of laparoscopic Rouxen-Y gastric bypass and laparoscopic sleeve gastrectomy: a prospective randomized trial. Ann Surg. 2009;250:234-41.

23. Skrekas G, Lapatsanis D, Stafyla V, et al. One year after laparoscopic "tight" sleeve gastrectomy: technique and outcome. Obes Surg. 2008;18:810-3.

24. Frezza EE, Reddy S, Gee LL, et al. Complications after sleeve gastrectomy for morbid obesity. Obes Surg. 2009;19:684-7.

25. Yo LS, Consten EC, Quarles van Ufford HM, et al. Buttressing of the staple line in gastrointestinal anastomoses: overview of new technology designed to reduce perioperative complications. Dig Surg. 2006;23:283-91.

26. Angrisani L, Cutolo PP, Buchwald JN, et al. Laparoscopic reinforced sleeve gastrectomy: early results and complications. Obes Surg. 2011;21:783-93.

27. Armstrong J, O'Malley SP. Outcomes of sleeve gastrectomy for morbid obesity: a safe and effective procedure? Int J Surg. 2010;8:69-71.

28. Tagaya N, Kasama K, Kikkawa R, et al. Experience with laparoscopic sleeve gastrectomy for morbid versus super morbid obesity. Obes Surg. 2009;19:1371-6.

29. Tichansky DS, Demaria EJ, Fernandez AZ, et al. Postoperative complications are not increased in super-super obese patients who undergo laparoscopic Roux-en-Y gastric bypass. Surg Endosc. 2005;19:939-41.

30. Magro DO, Geloneze B, Delfini R, et al. Long-term weight regain after gastric bypass: a 5-year prospective study. Obes Surg. 2008;18(6):648-51. 